\title{
Metaphysics, Bullshit, and the Analysis of Philosophical Problems
}

\author{
Bryan Frances \\ United Arab Emirates University \\ Forthcoming in Synthese
}

\begin{abstract}
Although metaphysics has made an impressive comeback over the past half century, there are still a great many philosophers today who think it is bullshit, under numerous precisifications of 'That's just bullshit' so that it's a negative assessment and doesn't apply to most philosophy (so it singles out metaphysics as particularly worse off than most other fields of philosophy). One encounters this attitude countless times in casual conversations, social media, and occasionally in print (e.g. Ladyman and Ross, 2007). Is it true?
\end{abstract}

What would suffice to show that metaphysics is not bullshit?

Here is one answer: show that a large and diverse group of metaphysical problems aren't bullshit. Then their good status would buttress metaphysics itself, at least to a significant degree. But what would suffice to show that a particular metaphysical problem isn't bullshit?

An answer: show that there are no tame, dismissive solutions to the problem. ${ }^{1}$ And we could show that there are no tame, dismissive solutions if we could offer an analysis of the metaphysical problem that showed that the problem itself forces one to embrace highly counterintuitive claims no matter what position one takes on it, assuming one doesn't fool around but makes the effort to offer a thorough response. Hence, if we can prove that a given metaphysical problem forces one to accept highly counterintuitive claims no matter what position one takes on it, then we will have shown that that problem is not bullshit. And if we can show that a large and diverse group of metaphysical problems satisfy that antecedent, then we will have shown that metaphysics itself isn't bullshit, to a significant degree. Can it be done?

In answering that question we will construct a particularly profitable way to analyze a philosophical problem.

The Problem of the Many is a typical metaphysical problem in that reflection on it generates bizarre philosophical views, such as the view that one thing can be numerically identical with many things, or the view that there are millions of ordinary trees where common sense says there is just one tree. One could easily suspect that it is a good example of a bullshit metaphysical problem. Anyone who talks to a diverse group of philosophers has heard the BS complaint many, many times.

\footnotetext{
${ }^{1}$ Previous attempts along this line include Schwitzgebel 2014, Frances 2019, and Frances 2021.
} 
Consider the following perspicuous rendition of the Problem of the Many:

$\mathrm{C}_{1}$ : There is at least one tree in my backyard.

$\mathrm{C}_{2}$ : Every tree in my backyard is exhaustively (not partially) composed of chemical atoms.

$C_{3}$ : For every tree in my backyard that is exhaustively composed of chemical atoms (at a time), there is a group of chemical atoms that composes it (at that time). ${ }^{2}$ (Some would say, with reason, that there is exactly one such group; $\mathrm{C}_{3}$ is more cautious.)

$\mathrm{C}_{4}$ : For every group of chemical atoms that composes a tree in my backyard, there is a group of chemical atoms with at least some distinct chemical atoms that composes a tree in my backyard. (The large groups (about $10^{30}$ chemical atoms in each) in question are literally about 99.9999999999999999999999999999\% overlapping and are, to all appearances, equally good candidates for composing a tree.)

$C_{5}$ : No tree in my backyard is exhaustively composed of distinctly-membered (in chemical atoms) groups of chemical atoms.

$\mathrm{C}_{6}: \quad$ There is at most one tree in my backyard.

On the face of it, the Cs are collectively inconsistent, since one can easily derive ${ }^{\sim} \mathrm{C}_{6}$ from the first five $\mathrm{Cs}$ using simple inferences (more on that below).

This essay is not concerned with the solutions to the Problem of the Many or any other classic metaphysical problem. I don't care what your favored solution is, and it won't matter to my arguments at all. Instead, we are focusing on the philosophically significant consequences of these problems themselves, not specific proposed solutions to them.

There are exactly eight possibilities with regard to the $\mathrm{Cs}, \mathrm{P}_{1}-\mathrm{P}_{8}$ : the first six collectively cover all the ways the conjunction of the Cs can be false, and the last two cover the two ways it can be true.

$$
\begin{aligned}
& \mathrm{P}_{1}: \sim \mathrm{C}_{1} \\
& \mathrm{P}_{2}: \sim \mathrm{C}_{2} \\
& P_{3}: \sim C_{3} \\
& \mathrm{P}_{4}: \sim \mathrm{C}_{4} \\
& P_{5}: \sim \mathrm{C}_{5} \\
& \mathrm{P}_{6}: \sim \mathrm{C}_{6} \\
& P_{7}: C_{1} \& C_{2} \& C_{3} \& C_{4} \& C_{5} \& C_{6} \& \text { there is no truth-preserving derivation to a contradiction } \\
& P_{8}: C_{1} \& C_{2} \& C_{3} \& C_{4} \& C_{5} \& C_{6} \& \text { there is a truth-preserving derivation to a contradiction }
\end{aligned}
$$

The reader can verify with mere sentential logic that the disjunction of the eight Ps is true. Thus, the disjunction of the eight Ps is logically true.

Let's examine only the obvious, immediate philosophical consequences of each disjunct, $\mathrm{P}_{1}-\mathrm{P}_{8}$. I will not be arguing that any particular disjunct is true (or not true). Instead, my commentaries on the disjuncts are meant to prove that each disjunct is philosophically counterintuitive, pretty much no matter how one

\footnotetext{
${ }^{2}$ All references to composition are to a particular moment and to chemical, not mereological, atoms.
} 
reasonably makes that notion precise. One can precisify it this way: a claim is philosophically counterintuitive at a time if and only if ( $i$ ) if the claim is true, then a great many of our ordinary beliefs and/or a significant portion of our most confidently held ordinary beliefs or belief-dispositions are false, or (ii) if the claim is true, then key philosophical ideas held by a large portion of philosophers at that time are false. Thus put, 'philosophically counterintuitive' might not be the best term. 'Philosophically significant' and 'philosophically consequential' were considered. Set aside aptness of vocabulary.

$\mathrm{P}_{1}$ : It's not the case that there is at least one tree in my backyard.

It might seem obvious that $P_{1}$ is philosophically counterintuitive. Any ordinary person or botanist would swear on their life that there is a tree in my backyard; so, $\mathrm{P}_{1}$ is about as counterintuitive as a claim can get. If $P_{1}$ is true, then due to the great number and variety of Problem of the Many applications, this will mean that an incredible number and variety of commonsensical claims aren't true (e.g. there won't be any people or cars either). Hence, if there aren't any trees in my backyard, then a great many of our ordinary beliefs, or a significant portion of our most confidently held ordinary beliefs or belief-dispositions, are not true.

There is, however, another possibility. Suppose $\mathrm{P}_{1}$ is true in this article, so 'There is at least one tree in my backyard' isn't true as that sentence is used in this work of philosophy. Even so, perhaps tokens of that sentence type are true when used in ordinary discourse. And if $P_{1}$ is true in ordinary discourse, then its being false in philosophical discourse may not be nearly as counterintuitive; in addition, it won't follow, at least immediately, that a great many of our ordinary beliefs, or a significant portion of our most confidently held ordinary beliefs or belief-dispositions, are not true.

This type of situation is not unheard of. For instance, everyday discourse employing 'miracle', 'conscious', 'believe', and 'justified' might not match up with philosophical discourse using the same terms, since the two discourses often (not always) employ different relevant meanings (i.e. ones that change truth-value). The following sentences, appearing in philosophical discourse, should raise a red flag:

If her belief was not justified, then 'Her belief was justified' isn't true in ordinary discourse.

If there are no miracles, then 'There are miracles' isn't true in ordinary discourse.

When I argued above that $\mathrm{P}_{1}$ is philosophically counterintuitive, it's at least arguable that I tacitly employed a similar premise in my argument:

1. If $P_{1}$ is true, then 'It's not the case that there is at least one tree in my backyard' is true in the discourse I am using right now in this article.

2. If that sentence is true in the discourse I'm using right now in this article, then it's true in ordinary discourse.

3. If it's true in ordinary discourse, then a great many of our ordinary beliefs, or a significant portion of our most confidently held ordinary beliefs or belief-dispositions, are not true.

4. Hence, by (1)-(3) if $P_{1}$ is true, then a great many of our ordinary beliefs, or a significant portion of our most confidently held ordinary beliefs or belief-dispositions, are not true. 
Sure enough, if premise (2) is true, then $P_{1}$ is philosophically counterintuitive (since (1) and (3) are true and $\{(1)-(3)\}$ entails (4), as the conditionals are material). But is (2) true?

There are five good reasons for thinking that (2) is true. First, there aren't any problematic terms here similar to 'miracle' or 'justified'; hence, that particular reason to be suspicious of (2) fails to apply. Second, I am explicitly saying - right now, if you like - that I am using, in this article, ordinary English-unless, of course, I supplement it with philosophical jargon, which clearly doesn't appear in the Cs. Third, there are good reasons, offered by Timothy Williamson (forthcoming; cf. van Inwagen, 2014), for thinking that philosophers are using ordinary English in their discussions of the ontology of the everyday world. Fourth, even if there is a language typically used in the "ontology room" that makes 'There are trees' express a proposition truth-conditionally different from the one it expresses in ordinary English, I am explicitly saying I'm not using that philosophical language and I am using ordinary English-and such a proclamation should shift the linguistic interpretation as appropriate. Fifth, there doesn't seem to be anything preventing me from using 'There is at least one tree in my backyard' with its ordinary meaning. In short, there is little good reason to think (2) isn't true, and there is excellent reason to think it is true.

But what if I simply am unable, in this article, to use ordinary English in my analysis of the Cs no matter what I say or do? Amazingly enough, it doesn't matter! Suppose the arguments of the previous paragraph are unsound and (2) isn't true, so 'There is at least one tree in my backyard' has different truth-values in ordinary discourse and in this article, despite the fact that $C_{1}$ is a sentence of perfectly ordinary English (however one wants to spell that out) and I have insisted that I am using ordinary English. Well, that would mean that we have been wildly wrong about linguistic interpretation-a philosophically counterintuitive result.

Hence, I am not claiming or even faintly suggesting that (2) is true, although that's what I suspect is the case. Instead, I'm saying that we have a philosophically counterintuitive result whether or not (2) is true.

In sum, here's my argument, with symbolization guidance regarding the entailments:

a. If $\mathrm{P}_{1}$ is true in ordinary discourse, then a great many of our ordinary beliefs and/or a significant portion of our most confidently held ordinary beliefs or belief-dispositions are false. $(A \supset Z 1)$

b. If (i) $P_{1}$ is not true in ordinary discourse but (ii) $P_{1}$ is true in this article, (iii) I have instructed that tokens of $\mathrm{P}_{1}$ in this article belong to ordinary English (even if they could belong to another language), and (iv) $\mathrm{P}_{1}$ does not contain any philosophical jargon not in ordinary English, then key philosophical ideas held by many contemporary philosophers are false. [( A \& B \& C \& D) D Z2]

c. I have instructed that tokens of $P_{1}$ in this article belong to ordinary English (even if they could belong to another language), and $\mathrm{P}_{1}$ does not contain any philosophical jargon not in ordinary English. (C \& D)

d. Hence, by (a)-(c), if $P_{1}$ is true in this article, then either (i) a great many of our ordinary beliefs and/or a significant portion of our most confidently held ordinary beliefs or beliefdispositions are false, or (ii) key philosophical ideas held by many contemporary philosophers are false. $\mathrm{B} \supset(\mathrm{Z1} \vee \mathrm{Z2})$ 
e. Hence, by (d) and the definition of 'philosophically counterintuitive', $P_{1}$ is philosophically counterintuitive.

We will encounter other premises like (2). Each has the form 'If ' $S$ ' is true in the discourse I'm using right now, then it's true in ordinary discourse'. I will call them bridge premises since they purport to bridge the alleged gap between my sentences in this article and ordinary discourse. The philosophical discourse need not belong to ontology, as other paradoxes come from other areas of metaphysics as well as other subfields of philosophy.

$\mathrm{P}_{2}$ : It's not the case that every tree in my backyard is exhaustively composed of chemical atoms.

If $P_{2}$ is true in this article, then 'It's not the case that every tree in my backyard is exhaustively composed of chemical atoms' is true in this article. But if that is true in this article, then it's true in ordinary discourse. If it's true in ordinary discourse, then we have a philosophically counterintuitive result, as any scientifically informed person could tell you. And if it's not true in ordinary discourse, because the relevant bridge premise is false, we still have a philosophically counterintuitive result for the same reason we had one in the case of $P_{1}$.

$P_{3}$ : It's not the case that for every tree in my backyard that is exhaustively composed of chemical atoms, there is a group of chemical atoms that composes it.

If $P_{3}$ is true in this article, then a tree in my backyard is exhaustively composed of chemical atoms but there is no group of atoms that composes it. If so, that is philosophically counterintuitive, as it goes firmly against key philosophical ideas held by many philosophers, if not natural scientists. A toy airplane is composed of Legos and there is a group of Legos that composes it (those 345 Legos that were in the box from the store); whereas a tree is composed of atoms but there is no group of atoms that composes it. That's the oddity.

$\mathrm{P}_{4}$ : It's not the case that for every group of chemical atoms that composes a tree in my backyard, there is a group of chemical atoms with at least some distinct chemical atoms that composes a tree in my backyard.

If $\mathrm{P}_{4}$ is true in this article, then there is a group of chemical atoms, $\mathrm{G}$, that composes a tree in my backyard-call that tree $T_{G}$-but there is no distinctly-membered group of atoms that composes a tree in my backyard. Clearly, $\mathrm{G}$ has got to have something like $10^{30}$ chemical atoms (give or take a few orders of magnitude) in order to compose a tree in my backyard, as atoms are very small compared to ordinary

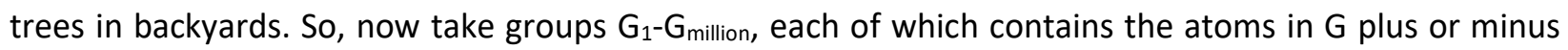
exactly one borderline case chemical atom. Given the way ordinary trees are, with enormous numbers of borderline cases of atoms, it's clear that these million $\mathrm{Gs}$ exist provided $\mathrm{G}$ exists. If $\mathrm{P}_{4}$ is true, then none of $\mathrm{G}_{1}-\mathrm{G}_{\text {million }}$ compose a tree in my backyard. Hence, there are over a million perfectly ordinary treeish groups of atoms that are literally 99.9999999999999999999999999999\% overlapping but only one of which composes a tree in my backyard.

$\mathrm{P}_{4}$ is not saying that those other $\mathrm{G}$ s fail to compose $\mathrm{T}_{G}$ specifically. Instead, it's saying that those other Gs fail to compose any tree at all (clearly, if they don't compose a tree in my backyard, they don't compose one elsewhere). Either those other Gs don't compose anything at all-but how is $G$ so 
magical compared to them that it composes something while they don't?-or they do compose things but those things aren't trees-but how could those things not be trees if the thing exhaustively composed by virtually identical $G$ is a tree? This means that a perfectly ordinary use of 'tree in my backyard' is stupefyingly, staggeringly precise, favoring $G$ over $\mathrm{G}_{1}-\mathrm{G}_{\text {million }}$ despite their virtual identity. But how on earth did 'is a tree in my backyard' acquire such an amazingly discriminating meaning? No one has offered even the faintest plausible story, and there is good reason to think none is forthcoming.

Obviously, one could offer a stipulation regarding 'is a tree in my backyard' so that under the resulting interpretation $\mathrm{P}_{4}$ is true and not philosophically counterintuitive. This would be similar to choosing a sharp cutoff for 'is rich'. However, that fact doesn't suggest for an instant that the sentence already, in the actual world prior to our possible stipulation, has an accurate interpretation with that consequence.

If you accept $\mathrm{P}_{4}$, then you surely have to wonder what shocking falsehoods there are about language and thought that you have hitherto presumed in all your work. Perhaps you have grown comfortable with accepting many other comparably outrageous epistemicist ideas, such as the idea that a perfectly ordinary token of 'The restaurant is a short walk from here' goes from true to not true in the space of a yoctometer $\left(10^{-24} \mathrm{~m}\right)$. It's great that you have managed to accept linguistic miracles: it's impressive that you have gotten over your previous discomfort with some radical claims. But then why think your continuing discomfort with any other radical claim isn't misguided as well?

There are two points here, worth distinguishing. First, there's the one is just made: if there can be over a million perfectly ordinary treeish groups of chemical atoms that are literally 99.9999999999999999999999999999\% overlapping but only one of which composes a tree in my backyard-if our ordinary words and thoughts have such incredibly exact meanings - then I could be wrong about a great many commonsensical claims about meaning.

Second, the grounds of meaning are made positively magical. Shifting over to the restaurant case for the sake of variety: who would have thought that if one puts all the factors that help fix linguistic meanings into one pot, that pot would give a perfectly ordinary utterance token of 'The restaurant is a short walk from here'-an utterance token made in certain highly specific circumstances, so we avoid speaker contextual issues - a truth condition that can distinguish two situations a yoctometer apart? If someone who used 'short walk' in the distant past had, counterfactually, farted during their utterance, or had a sore little toe that made him not want to make the walk to his favorite restaurant, would the true/untrue cutoff for the restaurant sentence token ended up at $10^{26}+117.11111 \mathrm{ym}$ instead of $10^{26}+117.11112 \mathrm{ym}$ ? No one has the slightest remotely plausible idea how perfectly ordinary token utterances of ordinary sentences such as those could ever acquire such utterly miraculous discriminating truth conditions. The alternative idea that these sharp cutoff facts are marvelous yet brute, so there is no pot of things that fix meanings, would show, once again, that we are wildly wrong about meaning and thought (there may be brute facts, but the sharp cutoff facts for tokens of 'There is a pumpkin by the tree' and 'The restaurant is a short walk from here' aren't among them).

To be sure, I'm not suggesting that $\mathrm{P}_{4}$ or any of the other seven disjuncts is false! (Regarding my commentary on $\mathrm{P}_{4}$, see Horgan, 1997 and Williamson, 1997a, 1997b.) There's no adequate argument 
here for or against epistemicism, dialethism, compositional nihilism, or anything else. I've argued only that $\mathrm{P}_{4}$ is philosophically counterintuitive since it is inconsistent with key philosophical ideas held by many contemporary philosophers.

$P_{5}$ : It's not the case that no tree in my backyard is exhaustively composed of distinctly-membered (in chemical atoms) groups of chemical atoms.

If $P_{5}$ is true in this article, then there are at least two distinctly-membered groups of atoms that both compose (at a time) the very same tree in my backyard. Since the groups differ in atom membership, there is chemical atom in one but not the other; call such an atom A. Hence, it appears that at a particular moment the tree is partially composed of $A$ and not partially composed of $A$. Unless we have made a truly fundamental error in how to think of composition at a time, we have a contradiction (millions of them, for other Problem-of-the-Many cases). In any case, the result is philosophically counterintuitive since it goes against key philosophical ideas held by many contemporary philosophers.

$\mathrm{P}_{6}$ : It's not the case that there is at most one tree in my backyard.

If $\mathrm{P}_{6}$ is true in this article, then there are at least two trees in my backyard-despite the fact that any nonphilosopher or botanist would swear on their life that there's just one tree there. I omit the standard comments on the relevant bridge premise.

$P_{7}: C_{1} \& C_{2} \& C_{3} \& C_{4} \& C_{5} \& C_{6} \&$ there is no truth-preserving derivation to a contradiction.

If you gaze at $C_{1}-C_{5}$, you'll be able to work out how to infer $\sim C_{6}$ from them using just four elementary inferences. If $\mathrm{P}_{7}$ is true, then at least one of the four is not truth-preserving (or the inference from $\mathrm{P}$ and $Q$ to $(P \& Q)$ isn't truth-preserving). Here are the four inferences:

11: From

$\mathrm{C}_{1}$ : There is at least one tree in my backyard

$\mathrm{C}_{2}$ : Every tree in my backyard is exhaustively composed of chemical atoms

To

There is at least one tree in my backyard that is exhaustively composed of chemical atoms.

12: From

There is at least one tree in my backyard that is exhaustively composed of chemical atoms

$\mathrm{C}_{3}$ : For every tree in my backyard that is exhaustively composed of chemical atoms, there is a group of chemical atoms that composes it

To

There is at least one group of chemical atoms that composes a tree in my backyard.

13: From

There is at least one group of chemical atoms that composes a tree in my backyard

$\mathrm{C}_{4}$ : For every group of chemical atoms that composes a tree in my backyard, there is a group of 
chemical atoms with at least some distinct chemical atoms that composes a tree in my backyard

To

There are groups of chemical atoms that are distinctly-membered (in chemical atoms) and that both compose trees in my backyard.

14: From

There are groups of chemical atoms that are distinctly-membered (in chemical atoms) and that both compose trees in my backyard

$\mathrm{C}_{5}$ : No tree in my backyard is exhaustively composed of distinctly-membered (in chemical atoms) groups of chemical atoms

To

$\sim \mathrm{C}_{6}$ : It is not the case that there is at most one tree in my backyard.

It is very difficult to see how any of these four inferences fails to be truth-preserving, which is what the truth of $\mathrm{P}_{7}$ requires. To say this is not to assume or imply that first-order logic can "capture" the logical form of each of $C_{1}-C_{6}$, whatever 'capture' means here. Even if first-order logic cannot "capture" at least one of the Cs, it would be philosophically counterintuitive to learn that one of I1-I4 isn't truth-preserving. The significance of $\mathrm{P}_{7}$ comes from its disjunctive consequences: either the inference-types are truthpreserving but we have badly misunderstood the meanings of the $\mathrm{C}$ sentences (so the rules are fine but don't apply to at least one of the Cs), or we have understood the meanings of the $C$ sentences but the inference-types aren't truth-preserving (the meanings are fine but the rules aren't). (Or both the rules and meanings aren't fine.) Hence, the truth of $\mathrm{P}_{7}$ would have profound implications for either (a) how we understand linguistic meaning, since the Cs are quite simple sentences but we don't understand them or (b) how we understand logic, since the inferences are quite simple and seemingly truth-preserving. And recall the point made earlier: there is an enormous number and variety of instances of the Problem of the Many, so (a) and (b) go far beyond the six Cs. Hence, $P_{7}$ is philosophically counterintuitive.

$P_{8}: C_{1} \& C_{2} \& C_{3} \& C_{4} \& C_{5} \& C_{6} \&$ there is a truth-preserving derivation to a contradiction.

If $P_{8}$ is true, then two things follow. First, $C_{6}$ isn't true (because $P_{8}$ says the obvious derivation to the contradiction ( $C_{6} \& \sim C_{6}$ ) goes through), which is philosophically counterintuitive for the reasons given for $P_{6}$. Second, $C_{6}$ is both true and not true, which is philosophically counterintuitive as well-especially since none of the $\mathrm{Cs}$ is even a paradoxical sentence, such as those from the Liar and other semantic paradoxes.

Since there is an enormous variety and number of cases that fit the Problem of the Many template, if we think the one l've used above regarding the tree in my backyard reveals a pair of true contradictory claims, then an enormous variety and number of other pairs of contradictory claims are true too (even if we adopt a paraconsistent logic, so that it's not the case that everything follows from just the contradiction in the backyard tree case).

My arguments regarding the obvious, immediate philosophical consequences of the individual P disjuncts, plus the logically true premise that at least one P disjunct is true, show that at least one of the following is both true and philosophically counterintuitive: 
- 'There are no trees' is true in ordinary discourse. (First disjunctive consequence of $\mathrm{P}_{1}$; the other disjunctive consequence, from the bridge premise, is covered in the last bullet point.)

- 'Trees aren't composed of chemical atoms' is true in ordinary discourse. (First disjunctive consequence of $\mathrm{P}_{2}$; the other disjunctive consequence, from the bridge premise, is covered in the last bullet point.)

- Trees are composed of chemical atoms but for a given tree there's no group of chemical atoms that composes it. $\left(P_{3}\right)$

- 'Tree in my backyard' (as well as an enormous number of similar phrases) is amazingly precise in the sense that there are there are over a million perfectly ordinary treeish groups of chemical atoms in my backyard that are literally $99.9999999999999999999999999999 \%$ overlapping but only one thing satisfies 'tree in my backyard'. $\left(\mathrm{P}_{4 .}\right)$

- At any given time, a tree is composed of multiple groups of chemical atoms that differ in atom membership (so the tree is composed of a group of chemical atoms that includes atom $\mathrm{X}$ but the tree is also, simultaneously, composed of a group of chemical atoms that fails to include $X)$. $\left(P_{5}\right.$.)

- 'There are at least two trees in my backyard' is true in ordinary discourse despite the fact that any non-philosopher or botanist would swear on their life that there's just one tree there. (First disjunctive consequence of $\mathrm{P}_{6}$ and $\mathrm{P}_{8}$; the other disjunctive consequence of $\mathrm{P}_{6}$ is covered in the last bullet point.)

- Some of the simplest and most certain inference rules aren't truth-preserving when applied to relatively simple, non-paradoxical sentences, so we have been wildly wrong about elementary logic; or we have little idea how to interpret an enormous number and variety of our ordinary, everyday, simple, non-paradoxical sentences. $\left(P_{7 .}\right)$

- There are a great many true contradictions coming from ordinary sentences (not weird, liartype ones), even assuming a paraconsistent logic. (Second disjunctive consequence of $\mathrm{P}_{8}$.)

- $P_{1}, P_{2}$, and $P_{6}$ are true in this article but false in ordinary discourse, despite not containing philosophical jargon and contrary to my author instructions. (Second disjunctive consequence of $P_{1}, P_{2}$, and $P_{6}$.)

If you insist that either (i) the disjunction of the Ps isn't true (despite having every appearance of a logical truth), or (ii) the disjunction of the above bullet points isn't true, that's fine: I still win, in the sense that the Problem of the Many forces one to take a philosophically counterintuitive view if one takes any serious view at all. That's because your view on the Problem of the Many, whether it's (i) or (ii), is highly philosophically counterintuitive, as characterized earlier. You might object: "No! Properly understood, my view is not counterintuitive". Fine: I still win, because the idea that your view, (i) or (ii), isn't wildly counterintuitive is itself wildly counterintuitive. No matter what you say about the Problem of the Many, you end up endorsing a philosophically counterintuitive view. You can of course just shut up and refuse to take any stand at all, but that's neither here nor there.

To have a vocabulary for generalizing beyond the Problem of the Many, I offer four useful stipulations 
(not claims).

Def: $R$ is philosophical paradox $=R$ is a plurality of claims $C_{1}-C_{n}$ such that upon consistent disambiguation (i) each $C_{i}$ comes from the same philosophical problem, (ii) each $C_{i}$ is individually highly plausible, (iii) it is highly plausible that there is a truth-preserving derivation from the Cs to a contradiction, and (iv) no proper sub-plurality of R satisfies (i)(iii).

I include the bit about disambiguation in order to make sure that a philosophical problem that fits the four conditions isn't a pseudo-problem. For example, the three sentences, 'We have mental processes', 'If something is mental, then it's not physical', and 'We are entirely physical' each have interpretations in non-philosophical contexts that are highly plausible. For the sake of a clean example, pretend that outside of philosophical discourse, occurrences of 'mental' sometimes (not always) have a truth-conditional contribution such that ' $x$ is mental' requires the truth of ' $x$ isn't physical' (so in this scenario 'mental' is unlike terms such as 'hydrogen', for which deference to experts is prevalent). Pretend also that in philosophical discourse 'mental' has no such connection to 'not physical'. The three sentences fail to generate a paradox because upon consistent disambiguation-so we interpret 'mental' univocally in the first two sentences-at least one of the Cs is highly contentious, to say the least. So, clause (ii) of the stipulation is not satisfied.

I add clause (iv) in an attempt to make sure R doesn't include anything irrelevant to the philosophical problem it is intended to capture (e.g. we should not add 'Trees are made of molecules' to $C_{1}-C_{6}$, since the six Cs are all that's needed to produce the derivation to a contradiction). As an alternative to (iv), we could just say "Do not include anything irrelevant in R".

I intend that many if not all of the traditional metaphysical paradoxes satisfy the stipulation's four conditions. This is part of the beauty of metaphysics: it's frequently the case that metaphysical problems are formulated in such a way as to satisfy (i)-(iv)-a feature that is not true of discourse on many other philosophical problems.

Def: $\mathrm{C}$ is a component of philosophical paradox $\mathrm{R}=\mathrm{C}$ is exactly one of $\mathrm{R}$.

Def: $\mathrm{X}$ is the Disjunctive Analysis, DA, of philosophical paradox $\mathrm{R}=\mathrm{X}$ is the disjunction of: the negation of each component of $\mathrm{R}$, the conjunction of the components of $\mathrm{R}$ plus the claim that there is a truth-preserving derivation from $\mathrm{R}$ to a contradiction, and the conjunction of the components of $\mathrm{R}$ plus the claim that there is no truth-preserving derivation from $\mathrm{R}$ to $\mathrm{a}$ contradiction.

For the Problem of the Many, the DA is the disjunction of the eight Ps. More generally, if there are $\mathrm{n}$ components of paradox $R$, then there are $(n+2)$ disjuncts of R's DA. DAs are disjunctive logical truths and serve as stepping stones to other key disjunctive truths:

Def: $\mathrm{X}$ is a Consequence Disjunction, $C D$, of philosophical paradox $\mathrm{R}=\mathrm{X}$ is a disjunction of obvious, immediate philosophically releveant consequences of all the disjuncts of the Disjunctive Analysis of R.

For the Problem of the Many, one CD is the disjunction of the nine bullet points preceding these four 
definitions. I can't think of any good reason for the idea that a given $\mathrm{R}$ must have just one $\mathrm{CD}$, although in order to be sure of the truth of the $C D$ we restrict ourselves to the obvious, immediate consequences of each disjunct of the corresponding DA (as illustrated by my arguments leading to the $\mathrm{CD}$ above).

Consider these five theses:

$\mathrm{T}_{1}$ : The Problem of the Many has a true Consequence Disjunction, given above.

$T_{2}$ : Each disjunct of that $C D$ is currently philosophically counterintuitive in the sense articulated earlier.

$\mathrm{T}_{3}$ : If a philosophical problem generates a $C D$ each disjunct of which is philosophically counterintuitive, then that problem is not bullshit.

$T_{4}$ : Many other metaphysical problems generate $C D$ disjunctions that make $T_{1}$ and $T_{2}$ true.

$\mathrm{T}_{5}$ : If $\mathrm{T}_{1}-\mathrm{T}_{4}$ are true, then metaphysics isn't bullshit.

My arguments regarding the obvious, immediate consequences of each of the Ps, with the conclusions of those arguments summarized in the $C D$, are clearly sound, as they were so unambitious. So, $T_{1}$ is true. Moreover, even a casual appreciation of the nine disjuncts of the $C D$ is enough to see that each is philosophically counterintuitive (this is a sociological fact, not a normative one). Hence, $T_{2}$ is true too.

Since $T_{3}$ is a universalized conditional, in order to cast doubt on it one has to make a strong case that there are bullshit philosophical problems that under consistent disambiguation have a CD each disjunct of which is currently philosophically counterintuitive. If my experience in philosophy is any guide, most BS philosophical problems once put in paradox form (which includes being stripped of relevant disambiguations) inevitably have a component claim that is highly contentious. Indeed, most BS philosophical problems are so hopeless that one cannot put them in paradox form at all.

One might object to $\mathrm{T}_{3}$ on the grounds that disputes about the CD disjuncts are "merely verbal". However, the arguments for the counterintuitiveness of the CD disjuncts addressed questions of interpretation, so platitudes about the prevalence of context dependence and other linguistic shenanigans are useless here. In addition, the use of 'merely' is crucial. Even if disputes about the CD disjuncts (all nine of them? Seriously?) are "verbal", that does not, at all, mean they are "merely verbal". Even if the disputes about each of the CD disjuncts were really about language in some deep sense, there isn't the slightest reason to think they all have the import of disputing 'Does that ridiculous drink count as a martini?' (cf. Bennett 2009).

In addition, thesis $\mathrm{T}_{4}$ (defended next) entails that there are a great many disjuncts, from CDs generated from other metaphysical problems. It's hardly plausible that the disputes about every disjunct from every metaphysical CD are merely verbal.

Regarding $\mathrm{T}_{4}$ : similar proofs of other true CDs can be constructed in the same fashion using materials from other metaphysical chestnuts such as the Statue-Clay problem, the Ship of Theseus problem, the Tibbles-Tib problem, van Inwagen's argument for incompatibilism, paradoxes about color tokens, 
the Sorites paradox, etc. This is not to say that the CD disjuncts in question are, in each case, as philosophically counterintuitive as those for the Problem of the Many. They aren't, but that hardly matters to the truth of $\mathrm{T}_{4}$. Further, some of those problems are a mixture of metaphysics and other fields such as the philosophy of language, mind, or logic, but that hardly matters either: many philosophical problems having nothing to do with metaphysics are a mix. If the philosophical significance of metaphysics lies mainly in the philosophy of language and logic, well, that is fine: that hardly suggests that metaphysics is bullshit.

Roughly put, what is needed to prove a surprising CD is this: a philosophy problem that under consistent disambiguation generates a set of highly intuitive claims such that when subject to highly intuitively truth-preserving inference rules, can be used to derive the negation of a highly intuitive claim. Those conditions are satisfied for problems in other fields of philosophy as well, thereby showing that those problems aren't bullshit either: Curry's Paradox, the Grelling-Nelson paradox, the No-No paradox, the Paradox of the Knower, Fitch's paradox, the Surprise Exam, the St. Petersburg paradox, the Suspense paradox, Russell's paradox, Yablo's paradox, the Dogmatism paradox, the Liar paradox, the Preface paradox, the argument for skepticism, the relativity of simultaneity paradox, etc.

One could object to $T_{5}$ on the grounds that the proofs of the CDs do nothing to solve any controversial philosophical problem. For instance, even after accepting the proofs we don't thereby come to know whether dialetheism is true, whether epistemicism is true, whether the simple inference rules in question are truth-preserving, whether trees are composed of chemical atoms, etc. I agree. But that fact fails to suggest that $T_{5}$ is false. The theses show that metaphysics isn't bullshit; they aren't intended to solve any philosophical problem other than the metaphilosophical one of 'Is metaphysics bullshit?'.

A much better objection to $T_{5}$ is that even though I'm right that there are a bunch of non-bullshit problems in metaphysics, much of its unique jargon is so ill-defined that many discussions using that term are hopelessly muddled. The key terms in the objection are 'much' and 'many', since using 'some' would hardly distinguish metaphysics from other areas of philosophy. To be clear, there is nothing in this article that rules out the possibility that the problem of jargon-muddle is significantly worse in metaphysics than other areas. However, the onus is on the critic to make the comparative point. That would be a large project.

Finally, I'm not merely saying that metaphysics has some philosophically important problems. I'm saying that (i) starting from several classic metaphysical problems we can construct (ii) proofs that (iii) establish the existence of philosophically counterintuitive truths-although the oddity, as we have seen, is that we aren't sure which philosophically counterintuitive disjuncts of the CDs are the true ones. That should go a long way in showing, by concrete examples, that metaphysics isn't bullshit. It might not be a shining city on a hill, filled with nothing but lovely, morally upstanding problems and issues, but it's not the slum many have accused it of being. Although I have not argued for the thesis here, I think that since metaphysics has (i)-(iii) going for it, and some other areas of philosophy do not (that's the bit I haven't argued for), metaphysics is, to a certain extent, further from being bullshit than those other areas of philosophy. Metaphysics deserves our admiration, and not our mere tolerance. 


\section{Bibliography}

Bennett, Karen. (2009). Composition, colocation, and metaontology. In Chalmers, Manley, and Wasserman (Eds.), Metametaphysics: new essays on the foundations of ontology. Oxford: OUP.

Frances, Bryan. (2021). Philosophical proofs against common sense. Analysis, 81, 18-26.

Frances, Bryan. (2019). The philosopher's doom: unreliable at truth or unreliable at logic. In Ted Poston and Kevin McCain (Eds.), The Mystery of Skepticism (pp. 187-199). Leiden: Brill.

Horgan, Terrance. (1997). Deep ignorance, brute supervenience, and the problem of the many. Philosophical Issues, 8, 229-236.

Ladyman, James and Don Ross. (2007). Every thing must go: metaphysics naturalized. Oxford: OUP.

Schwitzgebel, Eric. (2014). The crazyist metaphysics of mind. Australasian Journal of Philosophy, 92, 665-682.

Van Inwagen, Peter. (2014). Introduction: inside and outside the ontology room. In his Introduction to existence: essays in ontology. Cambridge: CUP.

Williamson, Timothy. (Forthcoming). Disagreement in metaphysics. In Maria Baghramian, J. Adam Carter, and Richard Rowland (Eds.), Routledge handbook of the philosophy of disagreement. London: Routledge.

Williamson, Timothy. (1997a). Imagination, stipulation, and vagueness. Philosophical Issues, 8, 215228.

Williamson, Timothy. (1997b). Replies to commentators: [Horgan, Gomez-Torrente, Tye]. Philosophical Issues, 8, 255-265. 